\title{
SPECIAL LECTURES
}


Indian Journal of Clinical Biochemistry, December 2007, Vol. 22 (Supl.)

SPL 1

Dr. S. Narayanan

Abstract Not Received

Indian Journal of Clinical Biochemistry, December 2007 
Indian Journal of Clinical Biochemistry, December 2007, Vol. 22 (Supl.)

SPL2

\title{
Pharmacogenetic: Should we be concerned?
}

\author{
Prof. Dr. Bhushan M. Kapur
}

Dept. of Clinical Pathology, Sunnybrook Health Scences Center, Department of Laboratory Medicine and Pathobiology, Faculty of Medicine, University of Toronto and Division of Clinical Pharmacology and Toxicology, The Hospital for Sick Children, Toronto.

The relatively new and emerging fields of pharmacogenetics and pharmacogenomics study how differences in individual genetic makeup affect the metabolism of drugs. Pharmacogenetics largely focuses on specific genes, such as drug-metabolizing enzymes. The most extensively studied genetic polymorphisms are those involving cyłochrome P450 2D6 (CYP2D6) CYP 2C19 and CYP3A4. A large number of drugs are substrates for these enzymes. Depending on the genetic makeup, there are poor metabolizers or ultra rapid metabolizer. Drug - drug interaction resulting by one drug inducing or inhibiting the metabolism of the other drug can also lead to unwanted clinical outcome. Measuring half-life and or patent/metabolite ratio is an indirect method to identify ppor metabolizer from ultra rapid metabolizer. In an unrecognized ultra-rapid metabolizer, almost all of the prescribed codeine converted to morphine leading to death of the infant who ws being breast fed. A patient who was on stable methadone dose suddenly became slow metabolizer because of the antihistamines prescribed to him.

\section{Curriculum Vitae:}

Dr. Bhushan M. Kapur, M.Phil, C.Chem, FRSC, FACB, FCAB.

Dr. Kapur graduated with a D.Phil from the University of Basel, Switzerland in 1967 under the guidance of Prof. Dr. T. Reichstein, (Nobel Laurent 1956). He joined the Addiction Research Foundation (ARF) in 1971 where he was the Director of Laboratories till May 1995. Since May 1995 he has ben with the Division of Clinical Pharmacology and Toxicology, The Hospital for Sick Children in Toronto as a scientist and consultant in toxicology. From August 2001 to November 2005 he took over the leadership of the toxicology department at St. Michael's Hospital in Toronto. He is staff member in the Department of Clinical Pathology, Sunnybook Health Sciences Center since October 2005. As a scientist, he has special interests are in drugs of abuse, markers of alcohol abuse and opiate dependency. Dr. Kapur has more than 200 scientific publications and presentations to his credit. His other credits include coauthoring a book titled Alcohol and the Identification of Alcoholics, invention and patent of an alcohol dipstick, a computer software CBAC: Computerized Blood Alcohol Calculator, and various book chapters on drugs of abuse. As ILO's (International Labour Organization, UN, Geneva) expert in Workplace Drug Testing, he has attended ILO sponsored Tripartite (Governments, Employers and Labour_meetings in Geneva and Oslo. Dr. Kapur is an Assistant Professor in the Department of Laboratory Medicine and Pathobiology, Faculty of Medicine, University of Toronto. He is a Fellow of The royal Society of Chemistry (UK), Academy of Clinical Biochemistry (USA) and Canadian Academy of Clinical Blochemistry.

E-mail: b.kapur@utoronto.ca 
Indian Journal of Clinical Biochemistry, December 2007, Vol. 22 (Supl.)

SPL3

\title{
Cryopreservation of human gametes and embryos in assisted reproductive technology.
}

\author{
Dandekar Sucheta ${ }^{1}$, Mangoli Ranjana', Mangoli Vijay², Desai Sadhana ${ }^{2}$ \\ ${ }^{1}$ GSS Medical College, Parel, Mumbai; ${ }^{2-}$ Fertility clinic and IVF centre, Mumbai
}

The last 3 decades have seen considerable improvements in the field of infertility. Assisted Reproductive Technology (ART) has emerged as a revolutionary treatment modality. Successful application of In Vitro Fertilization (IVF) was a major breakthrough in reproductive sciences. Along with IVF, cryopreservation gained importance in ART and became mandatory in many situations like freezing excess embryos obtained through IVF, preserving 'rare' sperms obtained from epididymal or testicular biopsies, or preserving gametes from patients undergoing cancer treatment. The present study involves studying effect of cryopreservation on 1- Male gametes for sperm function test and their fertilization potential. 2- Oocytes and embryos for retaining their fertilizing ability and growth potential in patients undergoing infertility treatments. Male gamete was used from following categories: 1: Normozoospermia $(n=25), 2$ : Oligozoospermia ( $n=25), 3$ : Asthenoteratozoospermia $(n=25)$, and 4: Obstructive azoospermia $(n=25)$. For the first three groups, sperms were frozen from the ejaculate. In case of obstructive azoospermia sperms were retrieved from the epididymis. For all the groups sperms were frozen using LN2 vapour freezing method. Thawing was done by single stage method. Thawed sperms were subjected to following Sperm Function Tests (SFT) Hypo Osmotic Swelling Test (HOST), Acrosome Reaction (AR), and Nuclear Chromatin Decondensation Test (NCDT). The thawed sperms were used to fertilize the oocytes by IVF and Intra Cytoplasmic Sperm Injection (ICSI) methods. Subsequent development of embryos and pregnancy rates was studied. Embryos were frozen using slow cooling method. After thawing those embryos with at least 50\% blastomeres intact were cultured for 24 hours before replacing back into the uterus. Oocytes were frozen using Vitrification method and after thawing, morphologically healthy, metaphase II oocytes were fertilized using ICSI technique. Subsequent fertilization and cleavage rates were evaluated. In Normozoospermia, thawed sperms showed no significant difference in HOST and NCDT. However, AR was negatively affected by $20 \%$. In other groups, HOST and AR were negatively affected whereas there was no significant difference in NCDT when compared to fresh samples. As compared to fresh sperms, Normozoospermia did not show significant difference in fertilization, cleavage, or pregnancy rate through IVF. All other groups showed significant decrease in fertilization rates with IVF. In all the study groups, ICSI significantly improved fertilization rate with thawed sperms. In case of embryo freezing, those frozen at zygote stage i.e 2 pronucleii showed higher survival and pregnancy rates, whereas, embryos frozen at grown cell stages resulted in lower survival rate. There was no significant difference in pregnancy rate in this category. Oocyte freezing resulted in $76.31 \%$ survival rate of which, $62.06 \%$ got fertilized. Embryo transfer resulted in $20 \%$ pregnancy rate. Cryopreservation has a definite beneficial effect in the outcome of ART. Sperm freezing, though useful in selected categories with IVF, proved to be very effective with the ICSI procedure. In unavoidable circumstances in patients undergoing cancer treatment, frozen samples can be considered as an effective backup. Embryo freezing resulted in increased cumulative pregnancy rate per cycle with obvious economical benefits to the patients. Oocyte freezing is still under developmental stage due to the unstable nature of chromosomal spindle at the unfertilized stage. However, the vitrification method has emerged as an effective alternative to other freezing techniques giving better results in terms of survival and fertilization after thawing. 
Indian Journal of Clinical Biochemistry, December 2007, Vol. 22 (Supl.)

\section{Curriculum Vitae:}

Dr. Sucheta P Dandekar

Dr. Sucheta Dandekar is Professor \& Head of the Department of Biochemistry at the Seth G.S. Medical College \& K.E.M. Hospital, Parel, Mumbai. She is also In-charge of the Central Clinical Biochemical Laboratories. She is presently holding charge of Ag. Deputy Director in National Plasma Fractionation Centre. She has to her credit three books and a number of publications in National and International Journals. She is a recognized post-graduate teacher and is an active member of the Medical Education Technology Cell. She has conducted as many as 21 programmes for MUHS. She is Assessor for National Accreditation Board for Testing and Calibration Laboratories (NABL). Dr Dandekar has started a certificate course in good laboratory practices. This is a very popular course. Dr. Dandekar has actively participated in all student related activities and is the convener of 'Shidori' programme and the 'parent teacher' programmes, both unique programmes at the G. S. Medical College. An active member of the Association Dr. Dandekar is recipient of the Seth G. S. Medical College \& K.E.M. Hospital Oration Award, which was given to her at the $32^{\text {nd }}$ Association of Clinical Biochemists of India Conference held at Patna. Dr Dandekar was given the Best Teacher Award (2006-07) by the Maharashtra University of Health Sciences. She has presented her research work at a number of national and international conferences. Dr. Dandekar as a visiting scientist at the University of Manitoba, Canada recently.

E-mail: suchetad@hotmail.com 
SPL4

\section{Partnerships for Progress: The APFBC, IFCC and National Associations of Clinical Biochemistry in the Asia-Pacific Region}

Joseph Lopez

President, APFCB and Member, IFCC Executive Board

The APFCB operates within a milieu of striking contrasts. Its geographical regions holds some of the world's richest and poorest countries with GDP per head of more than US $\$ 40,000$ and less than US $\$ 1,000$ respectively. It has two of the world's most populous countries which together hold about a third of the world's largest populations, both of which have some ofthe fastest economic growth rates in the world. The regional also contains some of the largest and smallest countries in the world. Inevitably, laboratory practices within the region range from the most sophisticated to the basic. The APFBC celebrate its Silver Jubilee in 2007 with a membership of 13 voting members associations, 14 corporate and an affiliate member. Its main activity is in education: besides the triennial Asian-Pacific congresses (the APCCBs), it organizes 3 types of visiting lecturships. Scientific activity is currently organized by members countries Japan, Taiwan and Australia. Yet, despite the surge in the activity level over the last 8 years, the APFCB's is yet to attract large areas of the region into its membership. All members of the APFCB are also IFCC members though the APFCB itself is not one. The only formal linkage between the two federations is the APFCB-IFCC agreement on the APCCB, that was signed in 2003. Nevertheless, the relationship between both federations has grown in recent years: the IFCC Visiting Lectureship programme with the reion is seen as an exemplary model of cooperation where IFCC provides and finances the lecturer, the APFCB arranges the itinerary and the national associations meet local expenses. Traditional healthcare delivery systems are evolving as governments int he region allow market forces to increase their influence. The concept of individual responsibility for healthcare is beginning to take hold. The APFCB and IFCC have a key role in the transfer of technologies and the maintenance of high quality standard for safe and effective patientcare within the region. Both federations will need to adapt to these changing paradigms.

(Note: the opinions expressed in this presentations are those of the speaker and do not necessarily represent those of the organizations in which he holds office)

\section{Curriculum Vitae:}

Joseph Lopez has been a clinical Biochemist for 34 years. He has been associated with the Asian and Pacific federation of Clinical Biochemistry since 1998 and became its president in 2004. Presently he is teaching clinical biochemistry at a private institute in Kaula Lumpur. He has carried out research in various aspectss of clinical chemistry and in particular spent many years studying the biochemical markers of primary liver cancer. His publications in local and international journals have covered the areas of methodology, quality assurance and application of tumour markers. He serves on the editorial board of the Indian Journal of Clinical Biochemistry and is an occaisonal invited reviewer of a number of journals in clinical chemistry. 
SPL 5

\section{Natural Antioxidants - A New Hope for Military and Civilian Applications}

Najmul Islam, Nazarul Hasan.

Department of Biochemistry, Faculty of Medicine, J. N. Medical College, A. M. U., Aligarh

Arrays of reasons contribute to the generation of free radicals in vivo, including oxidative stress, infections, etc. thereby resulting in disease proliferation. Far more troops have to be removed from field operations due to diseases than from war-related wounds. Troops who served in the Middle East came back home with Gulf War Syndrome. A mycobacterium is believed to have caused as many as 400,000 cases of Gulf War Syndrome. Because of antibiotic resistance (MDR-TB), antibiotic drugs as prophylaxis cannot be given. Thus, we explored the possible use of a natural antioxidant, namely allicin from garlic as a safe and economical adjunct in tuberculosis management. Blood of TB patients and healthy subjects were collected for PBMC isolation. Monocytes obtained from it were cultured and subjected to treatment with 0-500 ng/ $\mathrm{ml}$ of allicin for $24 \mathrm{hrs}$. Levels of TNF- $\alpha$ mRNA and MTB 85B mRNA was determined by RT-PCR. Intramonocyte glutathione (GSH) levels were also measured. Secreted IFN- $\gamma$ levels were assessed in TB monocyte culture supernatants using ELISA. We observed that allicin from garlic was a strong anti-oxidant and anti-inflammatory agent having beneficial effects on the immune system as well as in combating tuberculosis. We found that allicin from garlic helped in scavenging the free-radicals generated due to MTB-infection in human monocytes by suppressing TNF $\alpha$ mRNA and MTB 85B mRNA and ameliorating the levels of intramonocyte glutathione (GSH) and IFN- $\gamma$ levels. Allicin from garlic may act as a valuable anti-oxidant and anti-inflammatory agent that may combat TB. It is hoped that allicin may act as a potential stealth molecule helping both to military as well as civilians.

\section{Curriculum Vitae:}

Dr. Najmul Islam did his Ph.D. in Biochemistry in 1991. He did his post-doctoral training form AllMS, New Delhi and CWRU, USA. He is presently working as a reader in the dept. of Biochemistry, J.N. Medical College, A.M.U. Aligarh. He has got more than 50 press releases in International/National journals. He also has 94 internaitonal peer reviewed journals in which published papers have been cited and more than 200 citations in international journals. He possess 15 years of teaching experience.

E-mail: nx17@hotmail.com 
SPL6

\title{
Representative Study from Rural Andhra Pradesh on Adiposity and Meta- bolic Abnormalities
}

\author{
Shanthi Naidu', Krishnam Raju ${ }^{2}$, Clara Chow $^{3}$, Rama Raju $^{4}$
}

'Department of Laboratory Medicine - Biochemistry Division, CARE Hospital \& ${ }^{2}$ CARE Foundation, Hyderabad. ${ }^{3}$ George Instutite of International Health, Sydney, Australia ${ }^{4}$ Byrraju Foundation, Bheemavaram \& Hyderabad.

Data from rural India on metabolic parameters as a key risk factor to the phenomenon of cardiovascular disease is few, and hence 20 villages representative was considered and compared with the accepted Asian cut off's from recent studies. From 20 representative villages, 1085 individuals were considered from 4535 adults who underwent physical examination and finger prick glucose. Plasma glucose was measured by GOD-POD on ALFA Biotech PLD-951, creatinine and lipids were determined by Beckman Synchron clinical system with Beckman, Randox and Daichi reagents. The population of $18.4 \%$ of men and $26.3 \%$ of women were overweight rising to $32.4 \%$ of men and $41.4 \%$ of women if Asian definitions were used. Criteria for NCEP-ATPIII metabolic syndrome were met by $26.9 \%$ men and $18.4 \%$ of women with figures of $32.5 \%$ and $23.9 \%$ respectively if 'Asian' waist cut-off's were substituted. Dyslipidemia, adiposity and metabolic syndrome were common and significant to Asian studies, emphasizing metabolic risk factors to play a significant role in cardiovascular disease in this region.

\section{Curriculum Vitae:}

No CV 


\title{
Issues in Thrombophilia Investigation
}

\author{
A. Das Gupta, M.D., Ph.D., Fellow U.I.C.C. \\ Director Operations \& Head, Hematology \\ SRL Ranbaxy
}

It was approximately three decades back that our understanding of the molecular basis for familial clustering of venous thromboembolic events started to became clear. The role of naturally occurring anticoagulants such as Antithrombin, Protein $C$ and $S$ in neutralizing the procoagulant forces resulting from activation of clotting factors was better understood around this time. Abnormalities of these processes and of other related pathways were characterized and tests to detect such abnormalities were also developed. The terms "Thrombophilia" and "Hypercoagulable state" were coined to describe an "increased tendency to (venous) thrombosis" and the term "familial/inherited thrombophilia" was coined to describe thrombotic tendency resulting from inherited abnormalities of the procoagulant and anticoagulant pathways mentioned above. Progress in understanding of the molecular basis of thrombosis has resulted in a more appropriate definition of inherited thrombophilia: "Inherited thrombophilia is a genetically determined tendency to venous thromboembolism". Dominant abnormalities or combinations of less severe defects may be clinically apparent from early age of onset, frequent recurrence or family history. Milder traits may be discovered only by laboratory investigation. All genetic influences and their interaction are not yet understood.

\section{Curriculum Vitae:}

\section{Dr. Amar Das Gupta}

He did his Ph.D. in Biochemistry in April 1995, university of Mumbai, Mumbai. He is currently working as Executive Director - operations, SRL RANBAXY Clinical reference laboratories. He is the winner of many professional honours, scholarships, fellowships and awards. He is a member of many professional societies and has been the editor of Indian Journal of haematology and Blood transfusion for 3 years. He hhas served as a consultant of WHO to the govt. of myanmar has been empanelled as a assessor for National Board of accredition nominated Technical expert in haematology for NABL in 2005.

E-mail: amar_dspt@yahoo.co.in

Phone: (022) 27893416

Fax: (022) 66924717 


\title{
Association of MSP 1, 2 and Pfcrt genes with severe complications of Plasmodium falciparum malaria in children
}

\author{
Sudhanshu Shekhar Pati, Praveen Kishore Sahu, Radhanath Satpathy \\ Ispat General Hospital, Rourkela, Orissa.
}

Malaria is a major killer disease in the developing world. The pathogenesis of severe falciparum malaria is still not clearly understood. Moreover, there is no substantial data describing the correlation of specific parasite genotypes with malarial severity. Since the role of merozoite surface proteins (MSP-1 and 2) becomes crucial during the parasitic invasion to the host and manifestation of subsequent severe complications; there is a need to evaluate the correlation of these specific genotypes with severity of malaria. The prsent study was undertaken in a malaria endemic region, Sundergarh district of Orissa state, India. Genotypic analysis of MSP-1, MSP-2 and Pfcrt genes were carried out and their association with severity of malaria was determined. The major complications in the subjects were cerebral malaria and severe anemia; significantly associated with expression of genotypes RO $33(86.3 \%)$ and 3D7 $(95.8 \%)$ respectively. A high degree of multiclonicity $(\mathrm{MOI}=1.9)$ \& polymorphic behaviour of genotypes were observed in the study isolates. Overall Pfcrt gene mutation (K76T) was 95.2\%). In the present study RO 33 has emerged out as a genetic marker of severe malaria. The presence of drug resistance promotes complicated malaria in a locality is reconfirmed. Early detection of these genetic markers may predict the severe malaria to prevent morbidity and mortality.

\section{Curriculum Vitae:}

\section{Dr. Sudhanshu Shekhar Pati}

He has done M.D. in Biochemistry and is currently working as senior consultant and Head, department of Biochemistry, Ispat General Hospital, Rourkela. His area of interest is Molecular Pathology. He has published one paper in Indian Journal and a papers in international journals, and has written one chapter. He has been the Principal Investigator of 2 numbers of DBT funded projects on Malaria and Sickle Cell disease. He received the best paper awards twice in All India Steel Medical Conferences.

E-mail: sspati@rediffmail.com

Phone: 09437040542 
Indian Journal of Clinical Biochemistry, December 2007, Vol. 22 (Supl.)

SPL9

\section{Enymatic HbA}

Shigeki Uehara

Senior Manager International Marketing Department, Daiichi Pure Chemicals, Tokyo, Japan.

Manager International Marketing Department, Daiichi Pure Chemicals Tokyo, Japan

Diabetes mellitus has become a major health problem worldwide, reaching epidemic proportions in many developing countries with India being one major one. The high costs of diabetes are attributable to care for debilitating complications such as microvascular complications@predominantly retinopathy, nephropathy, and neuropathy and macrovascular complications, particularly stroke and coronary artery disease (CAD). Clinical studies proves that $\mathrm{HbAic}$ provides a retrospective index of the integrated plasma glucose values over an extended period and is not subject to the wide fluctuations observed when blood glucose levels are assayed. HbAic therefore is a valuable adjunct to blood glucose determinations in the assessment of glycemic control. HbAic is an indicator of confirmed diabetes mellitus and glycaemic control. There are many methods available to measure $\mathrm{HbAic}$. The selection of a method is influenced by several factors, including sample volume, patient population, dedicated instrument and cost. Recently, a rapid and simple enzymatic method for HbAic measurement using Fructosyl Peptlde Oxidase (FPOX) has been proposed and practically applied. This sophisticated technology uses an enzyme called as "Fructosyl Peptide Oxidase" (FPOX). FPOX is a novel enzyme having superior physicochemical properties such as stability under high temperature that makes it useful as an enzyme for clinical diagnosis. FPOX possess high selectivity for the detection of fructosyi valine and an excellent operational stability. Due to the excellent operational stability and perfect specificity, this method could be successfully applied to the assay of fructosyi peptides in the protease-digested blood samples. This technology is developed by Daiichi Pure Chemical, Tokyo, Japan. It was introduced globally in the AACC meeting of 2006. The Enzymatic HbAic using FPOX shows an excellent co-relation between HPLC yielding CV of 1.18 (low level specimen), 0.52 (middle level specimen) and 0.74 (high level specimen). Standardization is using JCCLS -CRM 004a is traceable to IFCC reference method. Moreover, there is no significant influence from potential serum interfering constituents and also from various hemoglobin variants and modified hemoglobins. Enzymatic $\mathrm{HbA}_{\text {ic }}$ has revolutionized the whole scenario of $\mathrm{HbAic}$ testing because of the benefits and the conveniences it offers. It can be easily adapted to any fully automated clinical chemistry analyzers, hence there is no separate instrument is involved. The total accuracy and precision offered by it has made it an acceptable and preferable methodology all over the world.

\section{Curriculum Vitae:}

Mr. Shigeki Uehara, Senior Manager International Marketing Department

Currently Working in Diagnostics Division, Daiichi Pure Chemicals Co. Ltd. 30 Years of experience in Japanese domestic and international diagnostic clinical chemistry market. Core responsibilities involved in marketing and product management. Responsible for Inian diagnostic business \& Scientific Project Development. 
Indian Journal of Clinical Biochemistry, December 2007, Vol. 22 (Supl.)

SPL10

\title{
Role of Vitamin D Suppmentation During Preeclampsia
}

\author{
Harbans Lal, Parul Singla and Smiti Nanda
}

Pt. B.D.Sharma Post Graduate Institue of Medical Sciences, Rohtak (Haryana)

Vitamin $D$ is unique amongst the various micronutrients due to its photobiogenesis and activation involving various organs. Its role in calcium homeostasis and prevention of skeletal disorders is well established. Recent studies have also implicated the role of vitamin D in certain physiological as well as pathological conditions such as growth and reproduction, carcinoma, psoriasis, etc. Some studies have also demonstrated that aberration in calcium homeostasis is responsible for hypertension during pregnancy. We have investigated the role of vitamin D supplementation in calcium homeostasis in preeclampsia. The study comprised of 200 primigravida women attending the antenatal clinic. One hundred of them were given the routine antenatal management (control group) while the other group of 100 women (vitamin D supplemented group) was also given vitamin D (33000 I.U., once every two weeks, between 28-36 weeks of gestation). Ten out of $100(10 \%)$ of the pregnant women were found to develop preeclampsia in the control group whereas the incidence of preeclampsia was found to be reduced to $4 \%$ in vitamin $D$ supplemented group. Their mean systolic blood pressure, at 36 weeks, was also found to be reduced to $122.0 \pm 0.73 \mathrm{mmHg}$ compared to $129.1 \pm 0.88 \mathrm{mmHg}$ in the control group. Vitamin D supplementation also showed a marked increase in serum calcium $(9.33 \pm 0.06$ verses $8.57 \pm 0.03 \mathrm{mg} \%$, in the control group. On the other hand, serum phosphorus $(3.06 \pm 0.05$ vs $3.63 \pm 0.04 \mathrm{mg} \%)$ and alkaline phosphatase $(7.32 \pm 0.15$ vs $7.88 \pm 0.17 \mathrm{KAU})$ levels were reduced. To assess the effect of vitamin $D$ supplementation on renal function and its correlation with calcium homeostasis, urinary calcium/creatinine ratio was calculated. The ratio was found to be significantly increased $(0.12 \pm 0.01$ vs. $0.09 \pm 0.01)$ on vitamin $D$ supplementation. It is suggested that Vitamin D should be supplemented during the third trimester of pregnancy, since it is efficacious both, in reducing the incidence of pregnancy-induced- hypertension as well as in restoring the disturbed calcium homeostasis in pregnant women particularly, those who are predisposed to preeclampsia.

\section{Curriculum Vitae:}

Prof. Harbans Lal has done Ph.D. and is currently working as senior professor of Biochemistry at PGIMS, Rohtak, Haryana. His areas of interest are Nutrition, clincial Biochemistry and ELISA. He has got 115 research papers published and has written 5 books. He is a member of many societies and possess WHO fellowship.

E-mail: lal_h@hotmail.com

Phone: 09416243615 
SPL1 1

\section{Identifying and Troubleshooting Common Interferences and Other Sources of Error in the TDM/tox Laboratory}

Amitava Dasgupta

Professor of Pathology and Laboratory Medicine, University of Texas Health Sciences Center at Houston

Serum concentrations of therapeutic drugs depend on many factors including the genetic @make-up of the patient. For example, polymorphism ofcytochrome $P 450$ mixed function oxidase greatly influence drug metabolism by an individual. In clinical laboratories, immunoassays are used for measuring drug levels because of ease of operation and fast turn around time. However, immunoassays have many limitations including cross-reactivity from compounds of similar structures especially metabolites of the parent drugs and other endogenous as well as exogenous compounds. Digoxin has a narrow therapeutic window 10.8 $1.8 \mathrm{ng} / \mathrm{ml}$ ) and because of high sensitivity of an immunoassay required for therapeutic drug monitoring ofdigoxin, many endogenous and exogenous compounds cross-react with digoxin immunoassays thus falsely increasing or decreasing true digoxin concentrations. Interference ofdigoxin-like immunoreactive factors (DLIS) in serum digoxin measurements by immunoassays is well documented in the literature. Although serum digoxin concentrations are falsely elevated due to elevated concentrations of DLIF found in volume expanded patients, elderly and new born babies, DLIF falsely lower digoxin values when measured by a microparticle enzyme immunoassay (MEIA: Digoxin II by Abbott Laboratories) $(1,2)$ This is a serious clinical problem because a clinician may increase digxoin dose based on low digoxin value thus putting the patient in a increased risk for digxoin toxicity. Howvere, new generation digoxin immunoassays are virtually free from such interferences (3). Moreover, DLIF are strongly bound to serum proteins and are virtually absent in protein free ultrafiltrates. Monitoring free digoxin also eliminates this interference. Steimer et al also reported serious negative interference of spironolactores and related drugs to the MEIA assay. One patient experienced digoxin toxicity due to increased digxoin dosage based on falsely low digoxin value as measured by the MEIA $(4,5)$. Various Chinese medicines and Indian Ayurvedic medicine Ashwagandha also interferes with polyclonal antibody based digoxin assays (6). Drugs metabolites often cross-react with immunoassays of parent drugs The cross-reactivity of carbamazepine 10, 11 -epoxide on carbamazepine immunoassays may vary from 0 to 95 (7) and there may be discordant between carbamazepine values obtained in two different clinical laboratories. More specific analytical technique such as high performance liquid chromatography is free from such interference. Hydroxyzine cross-reacts with PENTINA carbamazepine immunoassay(Dade Behring) (8) as well as immunoassay for tricyclic antidepressants (9). A less common occurrence is the interference ofheterophilic antibody with immunoassays for therapeutic drug monitoring. Moreover gross hemolysis, lipemia or hyperbilirubinemia may cause falsely elevated or decreased values when using immunoassays for determining therapeutic drug concentrations.

\section{Curriculum Vitae:}

Dr. Amitava Das Gupta has done his Ph.D. in a organic chemsitry, from stanford University, California in 1986. He is presently working as a Professor of Pathology and laboratory medicine, University of Texas, Houston Medical School. He is the winner of many honorary awards and is a member of many institutions and societies. He has been in the editorial board of many journals and has himself published more than 298 research papers. He has written 7 chapters in books.

E-mail: Amitava.dasgupta@uth.tmc.edu

Phone: 1 - (713) - $500-5407$

Fax: (713) - $500-0730$ 
Indian Journal of Clinical Biochemistry, December 2007, Vol. 22 (Supl.)

SPL 12

\title{
Clinical Practice of Therapeutic Drug Monitoring of Immunosuppressive Drugs
}

\author{
Dr. RK Sharma
}

Professor \& Head Department ofNephrology and Dean, Sanjay Gandhi Post Graduate Institute of Medical Sciences, Raebareli Road, Lucknow.

Therapeutic drug monitoring (TDM) is essential to maintain the efficacy of many immunosuppressive drug, while minimizing their side effects. TDM is critical for immunosuppressive drugs where dosing is concentration based to optimize their use. TDM has become more refined with the development of newer monitoring techniques and more specific assays. TDM has been useful for various immunosuppressive drug used in transplantation: cyclosporine, tacrolimus, mycophenolate mofetil, everolimus and sirolimus. TDM of cyclosporine A (CsA) has been well studied and CsA level monitoring 2 hrs after dosing $\mathrm{Ca}$ ) is more sensitive predictor of outcome than trough level $(\mathrm{Co})$. Trough level monitoring $(\mathrm{Co})$ is being used more widely for TDM of tacrolimus. Relationship for Co levels of tacrolimus and area under the curve $(A \cup C)$ has varied widely in clinical trials of TDM, with correlations ranging from 0.11 to 0.92 . Therapeutic drug monitoring for sirolimus (SRL) and everolimus is commonly done using Co levels. TDM ofmycophenolic acid (MPA) for mycophenolate mofetil (MMF) and enteric coated mycophenolate sodium (Myfortic) is evolving, rapidly with a trend for abbreviated AUC to monitor MPA drug concentration to arrive at correct dose of MMF or myfortic. 250 renal transplant patient on MMF and 75 on myfortic besides calcineurin inhibitor (CsA or TAC) and steroids as immunosuppressive drugs were evaluated at SGPGIMS, Lucknow. MPA-PK profiles were obtained at steady state AUC (0-12) hr and abbreviated AUC (0-4) were calculated in samples by EMIT method. Patients on enteric coated mycophenolate sodium (EC-MPS) demonstrated the typical pharmacokinetic profile of an enteric coated formulation with a delayed release of MPA, higher basal level of MPA and delayed peak, compared to patients on MMF although $12 \mathrm{hrs}$ AUC was same in EC-MPS) and MMF groups. AUC ranged from $30-60 \mathrm{mg} / \mathrm{hr} / \mathrm{L}$ abbreviated AUC 0-4 correlated well with the $12 \mathrm{hr} A U C$. Ci was the most useful single point, predicting absorption of EC-MPS. In a group of renal transplant recipients at our center (SGPGIMS), we compared cyclosporine A, Cz level monitoring and conventional Co monitoring. C2 monitoring improved the clinical outcomes for both denovo and maintenance renal transplant patients, reduced incidence and severity of acute rejection and unproved renal function in maintenance patients. In 126 renal transplant recipients change over from $\mathrm{Co}$ to $\mathrm{Cz}$ monitoring for CsA, demonstrated that 44 were under exposed to CsA in immediate post transplant period, and a significant number of patients on Co monitoring were over exposed to CsA in maintenance phase. Thus $\mathrm{Cz}$ monitoring would improve adequate $\mathrm{Cs} A$ dosing in immediate post transplant period and reduce CsA exposure in iater post transplant period. This would improve efficacy (immunosuppression) and reduce toxicity (nephrotoxicity) of CsA based on immunosuppression. Clinical therapeutic drug monitoring of immunosuppressive drugs which have a narrow therapeutic window and index is a useful tool for maximizing prevention of graft loss and organ rejection while it minimizes drug toxicity.

\section{Curriculum Vitae:}

Dr. Raj Kumar Sharma has done M.D. in Medicine and FAMS in nephrology. He is presetnly professor and head Nephrology, SGPGI, Lucknow. He is also the dean, SGPGI, Lucknow. He is the winner of many awards and honours. He is a member of many societies.

E-mail: rksharma@sgpgi.ac.in

Phone: 2668700 ext 2121 (off), 2122 (res)

Fax: 91-522-2668973, 2668017

Indian Journal of Clinical Biochemistry, December 2007 
SPL 13

\title{
Genetic polymorphism of CYP2C19 and its role in the eradication of H. pylori in North Indians
}

\author{
Krishan K Kohli
}

Department of Biochemistry, Postgraduate Institute of Medical Education and Research, Chandigarh.

This study was designed to establish the presence of genetic polymorphism of CYP2C19 and assess its role in the eradication of $H$. pyloriand healing of gastritis and oesophagitis in North Indians. Two hundred North Indians were phenotyped and genotyped for CYP2C19. The results demonstrated that CYP2C19 genetic polymorphism occurred with a frequency of $12 \%$ in healthy North Indians. CYP2C19*1, ${ }^{*}$ and * 3 demonstrated allele frequency of $0.7,0.3$ and 0 in healthy North Indians. CYP2C19* 2 accounted for $48 \%$ poor metabolizers (PMs) and 60\% defective CYP2C19 alleles. Concordance between the activity of OMZ hydroxylase and number of CYP2C 19 functional alleles was observed in healthy North Indians. Two hundred patients, $91 \mathrm{H}$. pylori positive and $109 \mathrm{H}$. pylori negative gastritis and/or oesophagitis were phenotyped and genotyped for CYP2C19. Genetic polymorphism of CYP2C19occurred a frequency of $12.5 \%$ and CYP2C19*1, * 2 and ${ }^{*} 3$ occurred with a frequency of $0.725,0.275$ and 0 in the patients. Ninety one $H$. pylori positive gastritis patients were treated with $20 \mathrm{mg}$ omeprazole (OPZ), $750 \mathrm{mg}$ amoxicillin (AMC) and $500 \mathrm{mg}$ tinidazole (TNZ) (bid) for 7 days. Extensive metabolizers (EMs) demonstrated $37 \%$ and PMs demonstrated $92 \%$ eradication of $H$. pylori. Noneradicated EMs were retreated with 40 $\mathrm{mg} \mathrm{OPZ} \mathrm{(bid)} \mathrm{and} 500 \mathrm{mg} \mathrm{AMC} \mathrm{(qid)} \mathrm{for} 14$ days. EMs after retreatment demonstrated $90 \%$ eradication. One hundred nine $H$. pylorinegative gastritis and/or oesophagitis patients were treated with $20 \mathrm{mg} O P Z$ bid for 28 days. EMs demonstrated $94 \%$ and PMs demonstrated $91 \%$ healing of gastritis, thus demonstrating no correlation between genetic polymorphism of CYP2C19 and cure of $H$. pylori negative gastritis and/ or oesophagitis. The study established the presence of CYP2C19 genetic polymorphism and its direct correlation with the eradication of $H$. pylori in North Indians. The results imply that if CYP2C19 genetic polymorphism is known prior to chemotherapy, an optimal dose of proton pump inhibitors can be prescribed for achieving better therapeutic outcome.

\section{Curriculum Vitae:}

Krishan Kumar Kohli has done Ph.D. in Medical Biochemistry from the university of Delhi in 1976. He is presently the Professor and head, department of Biochemistry, PGIMER, Chandigarh. He possess 24 years of teaching experience and 24 years of experience in clinical Biochemistry laboratory. he has 52 research papers published in journals of national and International repute. He has life membership of th National and 3 international scientific societies. he is awarded research projects by Indian Council of Medical research. He has participated in 50 national and international conference.

E-mail: kris_kohli2003@yahoo.com

Phone: 91-172-275 5175, 2755176 
Indian Journal of Clinical Biochemistry, December 2007, Vol. 22 (Supl.)

SPL 14

\title{
The use of an on-line inter-laboratory quality assurance programme: An Observation
}

\author{
Usha Anand, D.Vijaya, V.Aruna, C.V.Anand
}

PSG Institute of Medical Sciences \& Research, Coimbatore-641004.

To analyse the performance characteristics and utility of an inter-laboratory quality assurance programme. Two parameters, namely glucose and urea were chosen for this study.

Quality control samples of levels 1 and 2 obtained from Bio-Rad Laboratories were processed on Olympus AU400. The results obtained were used to develop two Levey-Jennings plots namely, LI and LI2. The mean and standard deviations for $L I$ were pre-assigned. $L 1$ was generated in the instrument using the results of the QC samples processed. L 2 was generated by entering the same values into an external software known as 'Unity Desktop', an on-line inter-laboratory quality assurance programme. The two graphs LJ1 and L2 were compared.

L 1- The mean +/-SD values $(\mathrm{mg} / \mathrm{dL}$ ) for levels 1 and 2 of glucose were $83+/-4.15$ and $273+/$ 13.75 respectively. The values for urea $(\mathrm{mg} / \mathrm{dL})$ were $35.9+/-2.1$ and $105.78+/-4.78$ for levels 1 and 2 respectively.

L 2- The mean +/- SD values for glucose were $81+/-0.85$ and $266.63+/-1.69$ for levels 1 and 2 respectively. For urea, the mean +/- SD were $35.85+/-0.91$ and $104.88+/-1.73$ for levels 1 and 2 respectively.

In several instances it was observed that the QC results showed up as an outlier in L2 while it was well within limits in LJ1.

In $L 1$ the mean value remains constant and the pre-assigned values that are used have a wide range. $L 2$ is a dynamic one and its mean and SD values are recalculated with every entry. If the results of the QC are accurate and reproducible, the SD becomes very narrow. Values well within two SD in LI become outliers in $L 2$.

To conclude, if the $L$ plot that is generated has values that have a very narrow scatter and hence a low SD, the false rejection rates might increase. 
Indian Journal of Clinical Biochemistry, December 2007, Vol. 22 (Supl.)

SPL 15

\title{
Analytical and Technical Evaluation of Lipid Profile and Glucose with Cholestech DX Point of Care Testing (POCT) Equipment
}

\author{
C.V.Anand, Usha Anand, D. Vijaya \& V Aruna \\ Department of Biochemistry, PSG Institute of Medical Sciences \& Research, Coimbatore
}

Centralization of laboratory diagnostics and an increasing number of urgent requests and non-standard samples raise the need for short turn-around times and high throughput analyzer for lipid profile tests. The aim of the present study was to evaluate the analytical and technical performance of Cholestech LDX.

The Cholestech LDX System combines enzymatic methodology and solid-phase technology to measure total cholesterol (TC),HDL-cholesterol(HDL-C), triglycerides(Trig)and glucose (Glu).

Whole blood from a fingerstick or venepuncture (collected in a lithium heparin-coated capillary tube). The sample (35 $\mathrm{L}$ ) is applied to a Cholestech LDX cassette. The cassette is then placed into the Cholestech LDX Analyzer. The analyzer has a unique system, which separates the plasma from the blood cells. A portion of the plasma flows to the right side of the cassette and is transferred to both the TC and Trig reaction pads. Simultaneously, plasma flows to the left side of the cassette where the LDL and VLDL are precipitated with a reagent containing dextran sulfate and magnesium acetate. The filtrate, containing both glu and HDL-C, is transferred to glu and HDL-C pads. The total analysis time is three minutes

Within-run precision, day-to-day precision of TC, HDL-C, Trig, LDL-C and Glu were determined. Results were also compared with Cobas Integra 400 Plus of Roche Diagnostics.

Cholestech LDX is a very reliable POCT for lipid profile and glucose testing. To our knowledge, this is the first evaluation of the analytical and technical performance of the POCT system. It is suited for the physician's office, in health camps and the corporate sector (master health check), enabling the physician to take up timely intervention. 